\title{
Analysis and Diagnosis of Coal Shearer Machine Fault Based on Improved Support Vector Theory
}

\author{
X. Zhang, X.M. Ma, Z.S. Yang \\ Electronic Control Institute of Xi'an University of Science and Technology \\ Xi'an, China
}

\begin{abstract}
In coal shearer monitoring system, the early detecting of fault is key technique for preventing the shearer fail. In this paper, the improved support vector machine theory is introduced to detected shearer fault under the mine underground, the improved algorithm based on support vector machine theory is analyzed, the multiple fault classifier is used to judge the fault types of coal shearer. The temperature fault types of coal shearer are reconstructed. Simulation results verify the validity of this method for early detecting fault under strong noise background in the coal shearer monitoring system.
\end{abstract}

Keywords-cool shearer; support vector machine; multiple fault diagnostics; diagnosis fault

\section{INTRODUCTION}

With the continuous development of mining mechanization, the current general trending is the development and popularization of coal mining group. And Shearer is one of the important equipment for production mechanization and modernization of coal mines, Safety and reliability of its operation directly affect coal mine production safety and economic benefits, however, due to limitations of the coal industry, shearer's work environment is often very vile,easily changed by the huge impact loads and coal dust, mist and other aspects of the contamination during operation. So launch Shearer failure diagnosis technology, not only to find fault, to prevent accidents, while reducing losses that caused by fault to improve coal production reliability, efficiency.So it is essential and necessary for Shearer fault to detect and diagnose.

In intelligent diagnostic methods fuzzy diagnosis, expert systems and artificial neural networks are used currently more commonly, often requires a lot of empirical knowledge or failure data samples, however in the field of mechanical fault diagnosis, get plenty of typical fault data samples is very difficult. In addition, these methods need to first digital signal processing to extract a Characteristic quantity of the signal, and for different fault in the end what features should be extracted and how to extract,there is no uniform provision, thus limiting the application of these intelligent diagnostic method. A new universal learning methods is developed on the basis of statistical learning theory----SVM, it can solve the problem of small sample study, and in the case of small samples can build a classifier better generalization ability. Support vector machine is machine learning methods that based on statistical creativity theory, The basic idea is that through a nonlinear transformation is defined by the inner product function of the input space transformed into a high-dimensional space, then seeking optimal classification face in this space,so support vector machine to solve the problem of high-dimensional. Support vector machine has a rigorous theoretical foundation, it uses structural risk minimization principle, has a good ability to promote. SVM is a algorithm of convex quadratic optimization problem, and the algorithm can guarante to find the global optimal solution, also can solve the practical problems of small sample, nonlinearity, high dimension, etc. Therefore, support vector machine is a hot issue in today's research, currently used in pattern recognition, regression analysis and other fields. In this paper, the theoretical is based on support vector machines, research the problem of shearer machine fault $[5,6]$.

\section{SHEARER FAULT CLASSIFICATION}

Shearer fault according to failure site can be divided into four parts: (1) mechanical fault; (2) hydraulic fault; (3) cool down and water supply systems; (4) electrical control fault. The shearer fault parameters block diagram is shown in Fig.1[3].

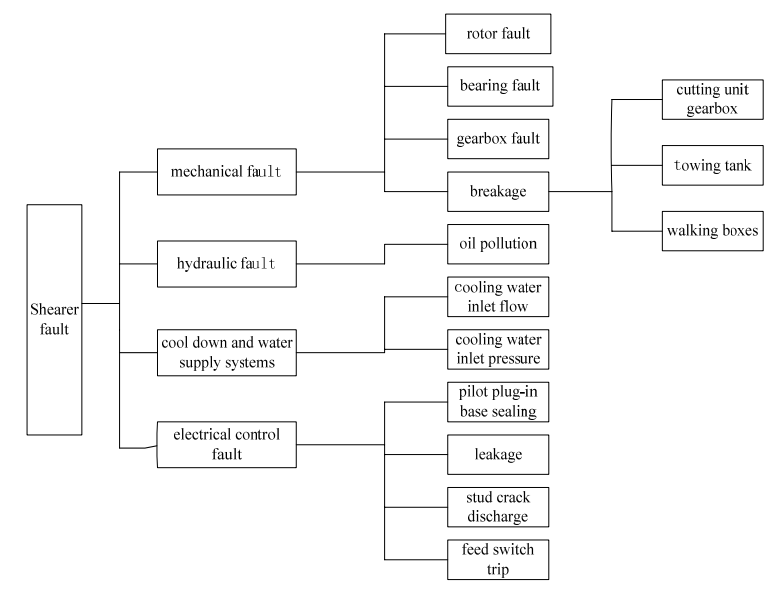

FIGURE I. SHEARER FAULT PARAMETERS BLOCK DIAGRAM

III. THEORY AND AlgORITHMS OF TWO

CLASSIFICATION SUPPORT VECTOR MACHINE

There are two types of set of training data samples

$$
\left(x_{i}, y_{i}\right), i=1,2, \cdots, n, x \in R^{d}, y \in\{+1,-1\}
$$

where $n$ is the number of training samples; $d$ is the dimension of each training sample vectors; $y$ is category 
label(its value is +1 for a class, -1 is for another class)

For nonlinear classification, The first to use a non-linear mapping, the data samples are mapped from the original space into a high dimensional feature space, Solving optimal classification in high-dimensional feature space. Dimension of high-dimensional feature space may be very high, however, support vector machine kernel function cleverly solved the problem. According to the theory of function, as long as an auditor culvert satisfy Mercer conditions, it corresponds to Some the inner product of transform space, such high-dimensional space is actually only need the inner product operation, and this inner product operation can use the function to achieve of the original space, without knowing the specific form of transformation. Therefore, the use of appropriate inner product function in optimal classification plane, then linear classification can be achieved after a nonlinear transformation, the computational complexity without increasing, category surface should satisfy the constraints,it can be written as follows:

$$
y_{i}\left(w \cdot \Phi\left(X_{i}\right)+b\right) \geq 1_{i=1,2, \cdots, n}
$$

where $w$ is Weights vector of classification surface; $b$ is classification threshold.

Taking into account some training samples can not be correctly classified by classification surface, therefore, introducing a slack variable, make the classification face constraints changes, the it can be shown as follows:

$$
y_{i}\left(w \cdot \Phi\left(X_{i}\right)+b\right) \geq 1_{-} \xi_{i}, i=1,2, \cdots, n ; \quad \xi_{i} \geq 0
$$

Those samples make equal sign of the formula (2) is established called Support Vector. The classification surface makes the classification interval maximize that is called optimal classification surface, the classification interval can be expressed as follows:

$$
2\left|y_{i}\left(w \cdot \Phi\left(X_{i}\right)+b\right)\right| /\|w\|=2 /\|w\|
$$

So the problem of constructing optimal classification surface is converted to request the minimum of the function under the formula (2) constraint, when determining the optimal classification, considering the minimum misclassification sample and maximum class interval, the constant control the degree of punishment of right or wrong sub-sample. This is a convex quadratic optimization problem, and can guarantee to find the global optimal solution of extreme Solution, Lagrange function can be used to make the original problem into simple dual problem: under the constraint conditions of

$$
\sum_{i=1}^{n} y_{i} \alpha_{\alpha \vee \delta} C \geq \alpha_{i} \geq 0 \quad i=1,2, \cdots, n,
$$

then solving the maximum value of the following function can be written:

$$
Q(\alpha)=\sum_{i=1}^{n} \alpha_{i}-\frac{1}{2} \sum_{i, j=1}^{n} \alpha_{i} \partial_{j} y_{i} y_{j} K\left(X_{i}, X_{j}\right)
$$

the optimization of the coefficient obtained by equation (3) must meet this function, it can be expressed as follows:

$$
\alpha_{i}\left(y_{i}\left(w \cdot \Phi\left(X_{i}\right)+b\right)-1+\xi_{i}\right)=0, i=1,2, \cdots, n
$$

Therefore, much value will be for 0 ; few values will not be 0 ,and those values will corresponding to those samples that make equal sign of the formula (2) is established, called Support Vector.Only those samples of Support Vector can determine the final classification results.

Using function (3) find the optimized coefficients, then for the given test sample, the general form of classification function in support vector machine classifier can be shown as follows:

$$
f(x)=\operatorname{sign}\left\{\sum_{S V M} \alpha_{\mathrm{i}} y_{i} K\left(x_{i}, x\right)+b\right\}
$$

where sign \{ \} represents sign function. In the positive and negative aspects of the classification function can determine the classification belongs to.

Choosing different the inner product kernel functions to form different algorithms, there are four kernel functions are more commonly useful in the classification: linear kernel, polynomial kernel function, RBF kernel function and Sigmoid kernel function[1].

\section{Multiple Fault Classifier}

Multi-Classification support vector machine algorithm is based on dichotomous mainly has two kinds: "one to one"and"one to many". This section respectively has a brief introduction.

"one to one"algorithm is put forward by Dr. S.Knerr, based on classification method of Class 2 problem, it can reduce "one to many" classification methods that can not be sub-regional. That in all n classes, then choosing two different categories to form a sub-classifiers every time, that is structuring all possible types classification in class samples, each of the two categories of the classifier is trained only with the class of Class 2 training samples, and using the "referendum law" to classify the test samples.

"One to many" algorithm was first proposed by Dr.Vapnik. This method constructs the $\mathrm{n}$ decision function, Each decision function will separate Class 1 from Other groups.

Combined with "one to one" and "One to many" algorithms,there are given a multi-value classifier. In the classification of the test data sample, first test data sample should be input single Classifier, when the output of decision function is 1 , it belongs to the objective function and no fault samples. Then end of the test. On the contrary, if the output of the function is -1 , was judged to be the failure data, then the data is input in the classifier the Category 1 . That 
structure the $\mathrm{n}$ classifiers in fault data, when in the $\mathrm{m}$-th classifier, The training set of the m-th class as a class, is denoted $y_{i=1}^{m}$, the remaining sample set is denoted $y_{i}^{m}$ $y_{i=-1}$, optimized, can establish classification function of the $\mathrm{m}$-th classifier, the function can be written as follows:

$$
f^{m}(x)=\operatorname{sign}\left\{\sum_{S V M} \alpha_{i}^{m} y_{i}^{m} K\left(X_{i}, x\right)+b^{m}\right\}
$$

If the output of discriminant $f^{1}(x)$ is 1 , the sample belongs to class 1 , the test is finished; otherwise, test samples will go in the classifier 2 automatically, then the output of discriminant $f^{2}(x)$ is 1 , the sample belongs to class 2, the test is finished, otherwise, test samples will go in the classifier 3 automatically. And so on, for classifier $k$, if If the output of discriminant $f^{k}(x)$ is 1 , the sample belongs to class $\mathrm{k}$, the output of the discriminant is -1 , the sample does not belong to the any classifier[2].As shown in figure 2.

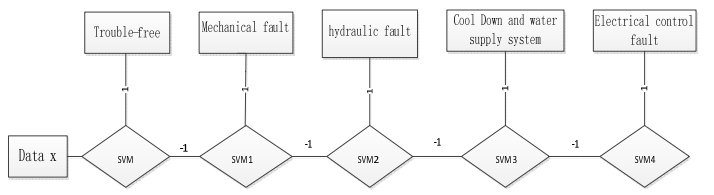

FIGURE II. THE FLOW DIAGRAM OF MULTI-FAULT CLASSIFIERS

Failure temperature datas of the four kinds of faults are sampled by a temperature sensor. Mechanical failure and hydraulic failure, for example, selecting 20 data samples as the study sample, each training data sample has 13 characteristic parameters, and using the Gauss kernel function(RBF), it is selected and as a classifier kernel to carry out fault classification[4]. As shown in figure 3.

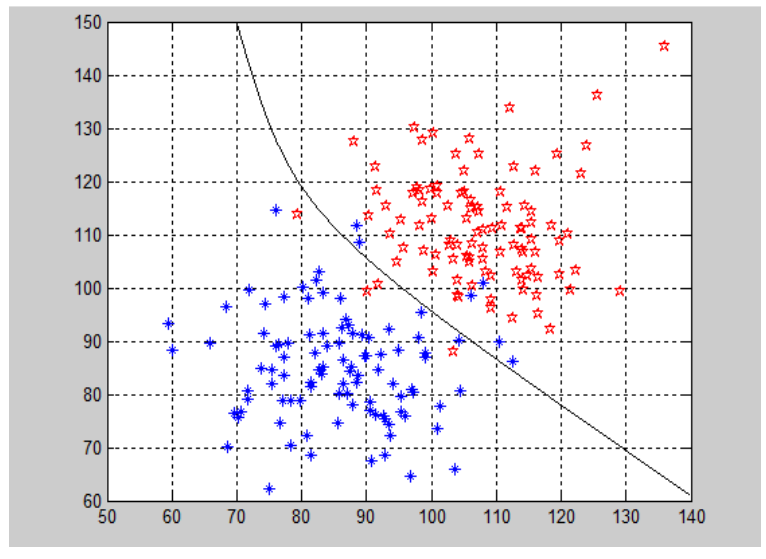

FIGURE III. TWO FAULT CLASSIFICATION RESULTS

Shearer troubleshooting includes a total of four kinds of fault types, in order to verify the correctness of multi-fault classifier, depicting three from each of data samples as the test data samples.The test results are shown in table 1 . And the type of data samples and classification results is consistent. Indicating that classification can properly classify a variety of fault.

\section{TABLE I. Multi-FAult TEST Results of SHEARER Fault BASED ON} SVM

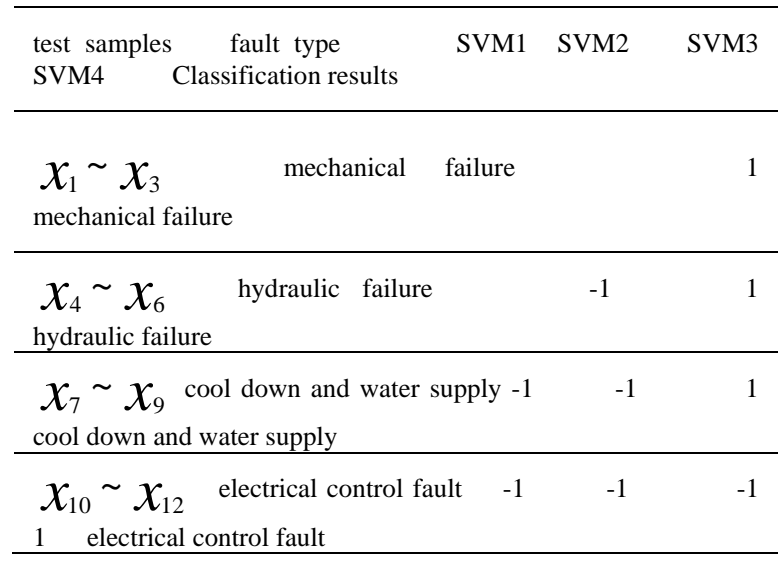

\section{V.CONCLUSION}

This paper discusses a method of fault diagnosis and classification of shearer that it based on support vector machine.Using multiple fault diagnosis method to diagnose shearer machine fault, and test samples of the data classifier only a few time-domain data samples, without prior extraction signal of characteristic quantities, and the algorithm is simple. In this paper, not only to use multi-fault classifier to diagnose shearer machine fault, but also is tested. As can be seen from the results of this diagnostic method in shearer failure has relatively high accuracy.

\section{REFERENCE}

[1] Zhousuo Zhang, Lingjun. Li \& Zhengjia He. multi-fault classifier and applications based on SVM.Mechanical Science and Technology[J]. School of Mechanical Engineering, Xi'an Jiaotong University, 2004,23(5):0538 0601.

[2] Chang lin Wang, Wei Lin, Hong bao. Chen, Qi mao Qin \& Yi mei Song. The study of mechanical multiple faults intelligent classification method based on Support Vector Machine[J]. Coal Mine Machinery,2009,30(10):0243 0245.

[3] Zengqiang Wang, Wanli Che, Zhenlin Quan, Xuhui Zhang \& Hongwei Ma. The study of line monitoring and fault diagnosis System of electric traction shearer [J].Heavy Machinery, 2012(5):41 44.

[4] Jingyi Du, Yuanbing Hou. The study of fault diagnosis theory and methods based on kernel methods[M].Beijing : Peking University Press, 2010.8

[5] Shengfa Yuan, Fulei Zhu. Support Vector Machine and Its Application in Machine Fault Diagnosis[J].Vibration and shock, 2007,26(11):29 58.

[6] Yajuan Liu, Xiaoqin Zhang. Fault diagnosis of rotating machinery based on support vector machine[J].Computer Engineering and Design,2005,26(12):3436 3438. 